J. Korean Math. Soc. 48 (2011), No. 3, pp. 551-563

DOI 10.4134/JKMS.2011.48.3.551

\title{
SIGNED TOTAL $k$-DOMATIC NUMBERS OF GRAPHS
}

\author{
Abdollah Khodkar and S. M. Sheikholeslami
}

\begin{abstract}
Let $k$ be a positive integer and let $G$ be a simple graph with vertex set $V(G)$. A function $f: V(G) \longrightarrow\{-1,1\}$ is called a signed total $k$-dominating function if $\sum_{u \in N(v)} f(u) \geq k$ for each vertex $v \in$ $V(G)$. A set $\left\{f_{1}, f_{2}, \ldots, f_{d}\right\}$ of signed total $k$-dominating functions of $G$ with the property that $\sum_{i=1}^{d} f_{i}(v) \leq 1$, for each $v \in V(G)$, is called a signed total $k$-dominating family (of functions) of $G$. The maximum number of functions in a signed total $k$-dominating family of $G$ is the signed total $k$-domatic number of $G$, denoted by $d_{k S}^{t}(G)$. In this note we initiate the study of the signed total $k$-domatic numbers of graphs and present some sharp upper bounds for this parameter. We also determine the signed total $k$-domatic numbers of complete graphs and complete bipartite graphs.
\end{abstract}

\section{Introduction}

In this paper, $G$ is a finite simple graph with vertex set $V(G)$ and edge set $E(G)$. For a vertex $v \in V(G)$, the open neighborhood $N(v)$ is the set $\{u \in V(G) \mid u v \in E(G)\}$ and the open neighborhood $N(S)$ of a set $S \subseteq V(G)$ is the set $\bigcup_{v \in S} N(v)$. The minimum degree of $G$, denoted by $\delta(G)$, is $\min \{|N(v)|$ | $v \in V(G)\}$. Consult [5] for the notation and terminology which are not defined here.

For a real-valued function $f: V(G) \longrightarrow \mathbb{R}$, the weight of $f$ is $w(f)=$ $\sum_{v \in V} f(v)$. For $S \subseteq V$, we define $f(S)=\sum_{v \in S} f(v)$. So $w(f)=f(V)$. Let $k \geq 1$ be an integer and let $G$ be a graph with $\delta(G) \geq k$. A signed total $k$-dominating function (STkDF) is a function $f: V(G) \rightarrow\{-1,1\}$ satisfying $\sum_{u \in N(v)} f(u) \geq k$ for every $v \in V(G)$. The minimum of the values of $\sum_{v \in V(G)} f(v)$, taken over all signed total $k$-dominating functions $f$, is called the signed total $k$-domination number and is denoted by $\gamma_{k S}^{t}(G)$. As assumption $\delta(G) \geq k$ is clearly necessary, we will always assume that when we discuss $\gamma_{k S}^{t}(G)$ all graphs involved satisfy $\delta(G) \geq k$. In the special case when $k=1$,

Received January 20, 2010; Revised April 8, 2010

2010 Mathematics Subject Classification. 05C69.

Key words and phrases. signed total $k$-domatic number, signed total $k$-dominating function, signed total $k$-domination number. 
$\gamma_{k S}^{t}(G)$ is the signed total domination number investigated in $[2,6]$. The signed total $k$-domination numbers of graphs was introduced by Wang [4].

A set $\left\{f_{1}, f_{2}, \ldots, f_{d}\right\}$ of signed total $k$-dominating functions on $G$ with $\sum_{i=1}^{d} f_{i}(v) \leq 1$, for each $v \in V(G)$, is called a signed total $k$-dominating family (STkD family) of $G$. The maximum number of functions in a signed total $k$-dominating family on $G$ is the signed total $k$-domatic number of $G$, denoted by $d_{k S}^{t}(G)$. The signed total $k$-domatic number is well-defined and $d_{k S}^{t}(G) \geq 1$ for all graphs $G$ in which $\delta(G) \geq k$, since the set consisting of any one STkD function forms a $\mathrm{STkD}$ family of $G$. A $d_{k S}^{t}$-family of a graph $G$ is a $\mathrm{STkD}$ family consists of $d_{k S}^{t}(G) \mathrm{STkD}$ functions. The signed total 1-domatic number $d_{1 S}^{t}(G)$ is the usual signed total domatic number $d_{S}^{t}(G)$, which was introduced by Henning in [3] and has been studied by several authors (see for example [1]).

In this note, we first study some basic properties of $d_{k S}^{t}(G)$ and find some sharp upper bounds for this parameter. Then we determine the signed total $k$-domatic numbers of complete graphs and of complete bipartite graphs, generalizing Propositions A and B.

We make use of the following results and observations in this paper.

Proposition A ([3]). If $G=K_{n}$ is the complete graph of order $n \geq 2$, then

$$
d_{S}^{t}\left(K_{n}\right)= \begin{cases}\left\lfloor\frac{n+1}{3}\right\rfloor-\left\lceil\frac{n}{3}\right\rceil+\left\lfloor\frac{n}{3}\right\rfloor & \text { if } n \text { is odd, } \\ \frac{n}{2}-\left\lceil\frac{n+2}{4}\right\rceil+\left\lfloor\frac{n+2}{4}\right\rfloor & \text { if } n \text { is even. }\end{cases}
$$

Proposition B ([3]). For $m \geq n \geq 1$,

$$
d_{S}^{t}\left(K_{m, n}\right)=\left\{\begin{array}{cl}
n & \text { if } n \text { and } m \text { are odd, } \\
\min \left\{n, \frac{m}{2}-\left\lceil\frac{m+2}{4}\right\rceil+\left\lfloor\frac{m+2}{4}\right\rfloor\right\} & \text { if } n \text { is odd and } m \text { is even, } \\
\frac{n}{2}-\left\lceil\frac{n+2}{4}\right\rceil+\left\lfloor\frac{n+2}{4}\right\rfloor & \text { if } n \text { is even. }
\end{array}\right.
$$

Observation 1. Let $G$ be a graph of order $n$ and $k \in\{n-2, n-1\}$. Then $\gamma_{k S}^{t}(G)=n$ and hence, $d_{k S}^{t}(G)=1$.

Observation 2. Let $G$ be a graph of order $n$. Then $\gamma_{k S}^{t}(G)=n$ if and only if $k \leq \delta(G) \leq k+1$ and for each $v \in V(G)$ there exists a vertex $u \in N(v)$ such that $\operatorname{deg}(u)=k$ or $\operatorname{deg}(u)=k+1$.

Proof. If $k \leq \delta(G) \leq k+1$ and for each $v \in V(G)$ there exists a vertex $u \in N(v)$ such that $\operatorname{deg}(u)=k$ or $\operatorname{deg}(u)=k+1$, then trivially $\gamma_{k S}^{t}(G)=n$.

Conversely, assume that $\gamma_{k S}^{t}(G)=n$. By assumption $k \leq \delta(G)$. Let, to the contrary, $\delta(G)>k+1$ or there exists a vertex $v \in V(G)$ such that $\operatorname{deg}(u) \geq k+2$ for each $u \in N(v)$. If $\delta(G)>k+1$, define $f: V(G) \rightarrow\{-1,1\}$ by $f(v)=-1$ for some fixed $v$ and $f(x)=1$ for $x \in V(G) \backslash\{v\}$. Obviously, $f$ is a signed total $k$-dominating function of $G$ with weight less than $n$, which is a contradiction. Thus $k \leq \delta(G) \leq k+1$. Now let $v \in V(G)$ and $\operatorname{deg}(u) \geq k+2$ for each $u \in N(v)$. 
Define $f: V(G) \rightarrow\{-1,1\}$ by $f(v)=-1$ and $f(x)=1$ for $x \in V(G) \backslash\{v\}$. Again, $f$ is a signed total $k$-dominating function of $G$, which is a contradiction. This completes the proof.

The following theorem generalizes the result on $\gamma_{k S}^{t}\left(K_{n, n}\right)$ obtained in [4].

Theorem 3. Let $k \geq 1$ be an integer. Then for every integers $m, n \geq k$,

$$
\gamma_{k S}^{t}\left(K_{m, n}\right)=\left\{\begin{array}{lll}
2 k & \text { if } \quad m \equiv n \equiv k \quad(\bmod 2) \\
2 k+1 & \text { if } \quad m \equiv k+1 \quad(\bmod 2), n \equiv k \quad(\bmod 2) \\
2 k+2 & \text { if } \quad m \equiv n \equiv k+1 \quad(\bmod 2) .
\end{array}\right.
$$

Proof. Let the partite sets of a $K_{m, n}$ be $X=\left\{x_{1}, x_{2}, \ldots, x_{m}\right\}$ and $Y=$ $\left\{y_{1}, y_{2}, \ldots, y_{n}\right\}$. We consider three cases.

Case 1. $m \equiv n \equiv k(\bmod 2)$. First note that if $f$ is a STkDF of $K_{m, n}$, then $\sum_{i=1}^{m} f\left(x_{i}\right) \geq k$ and $\sum_{i=1}^{n} f\left(y_{i}\right) \geq k$, which implies $\gamma_{k S}^{t}\left(K_{m, n}\right) \geq 2 k$. Label $\frac{m+k}{2}$ vertices of $X$ with +1 and the rest with -1 . Similarly, label $\frac{n+k}{2}$ vertices of $Y$ with +1 and the rest with -1 . It is clear that this labeling defines a STkDF, say $f$, of $K_{m, n}$. Since $f(N(v))=k$ for every vertex $v \in(X \cup Y)$, it follows that $\gamma_{k S}^{t}\left(K_{m, n}\right)=w(f)=2 k$.

Case 2. $m \equiv k+1(\bmod 2), n \equiv k(\bmod 2)$. First note that if $f$ is a STkDF of $K_{m, n}$, then $\sum_{i=1}^{m} f\left(x_{i}\right) \geq k+1$ and $\sum_{i=1}^{n} f\left(y_{i}\right) \geq k$ since $m \equiv k+1(\bmod$ $2)$. Label $\frac{m+k+1}{2}$ vertices of $X$ with +1 and the rest with -1 . Similarly, label $\frac{n+k}{2}$ vertices of $Y$ with +1 and the rest with -1 . It is clear that this labeling defines a STkDF, say $f$, of $K_{m, n}$. Since $f(N(y))=k+1$ for every vertex $y \in Y$ and $f(N(x))=k$ for every $x \in X$, it follows that $\gamma_{k S}^{t}\left(K_{m, n}\right)=w(f)=2 k+1$.

Case 3. $m \equiv n \equiv k+1(\bmod 2)$. First note that if $f$ is a STkDF of $K_{m, n}$, since $m \equiv n \equiv k+1(\bmod 2), \sum_{i=1}^{m} f\left(x_{i}\right) \geq k+1$ and $\sum_{i=1}^{n} f\left(y_{i}\right) \geq k+1$. Label $\frac{m+k+1}{2}$ vertices of $X$ with +1 and the rest with -1 . Similarly, label $\frac{n+k+1}{2}$ vertices of $Y$ with +1 and the rest with -1 . It is clear that this labeling defines a STkDF, say $f$, of $K_{m, n}$. Since $f(N(v))=k+1$ for every vertex $v \in(X \cup Y)$, it follows that $\gamma_{k S}^{t}\left(K_{m, n}\right)=w(f)=2 k+2$.

\section{Basic properties and upper bounds}

In this section we present basic properties of the signed total $k$-domatic number and find some sharp upper bounds for this parameter. Our first result is obtained by the definition of the signed total $k$-domatic number.

Theorem 4. Let $G$ be a graph of order $n$ and $\delta(G) \geq k>0$. Then $\gamma_{k S}^{t}(G)$. $d_{k S}^{t}(G) \leq n$. Moreover if $\gamma_{k S}^{t}(G) \cdot d_{k S}^{t}(G)=n$, then for each $d=d_{k S}^{t}$-family $\left\{f_{1}, \ldots, f_{d}\right\}$ of $G$ each function $f_{i}$ is a $\gamma_{k S}^{t}$-function and $\sum_{i=1}^{d} f_{i}(v)=1$ for all $v \in V$. 
Proof. Let $\left\{f_{1}, f_{2}, \ldots, f_{d}\right\}$ be a STkD family of $G$ such that $d=d_{k S}^{t}(G)$ and let $v \in V$. Then

$$
\begin{aligned}
d \cdot \gamma_{k S}^{t}(G) & =\sum_{i=1}^{d} \gamma_{k S}^{t}(G) \\
& \leq \sum_{i=1}^{d} \sum_{v \in V} f_{i}(v) \\
& =\sum_{v \in V} \sum_{i=1}^{d} f_{i}(v) \\
& \leq \sum_{v \in V} \\
& =n .
\end{aligned}
$$

If $\gamma_{k S}^{t}(G) \cdot d_{k S}^{t}(G)=n$, then the two inequalities occurring in the proof become equalities. Hence, for the $d_{k S}^{t}$-family $\left\{f_{1}, \ldots, f_{d}\right\}$ of $G$ and for each $i$, $\sum_{v \in V} f_{i}(v)=\gamma_{k S}^{t}(G)$, thus each function $f_{i}$ is a $\gamma_{k S}^{t}$-function, and $\sum_{i=1}^{d} f_{i}(v)=$ 1 for all $v$.

Corollary 5. If $G$ is a graph of order $n$, then $\gamma_{k S}^{t}(G)+d_{k S}^{t}(G) \leq n+1$.

Proof. By Theorem 4,

$$
\gamma_{k S}^{t}(G)+d_{k S}^{t}(G) \leq d_{k S}^{t}(G)+\frac{n}{d_{k S}^{t}(G)}
$$

Using the fact that the function $g(x)=x+n / x$ is decreasing for $1 \leq x \leq \sqrt{n}$ and increasing for $\sqrt{n} \leq x \leq n$, this inequality leads to the desired bound immediately.

Corollary 6. Let $G$ be a graph of order $n \geq 4$. If $2 \leq \gamma_{k S}^{t}(G) \leq n-1$, then

$$
\gamma_{k S}^{t}(G)+d_{k S}^{t}(G) \leq n
$$

Proof. Theorem 4 implies that

$$
\gamma_{k S}^{t}(G)+d_{k S}^{t}(G) \leq \gamma_{k S}^{t}(G)+\frac{n}{\gamma_{k S}^{t}(G)}
$$

If we define $x=\gamma_{k S}^{t}(G)$ and $g(x)=x+n / x$ for $x>0$, then because $2 \leq$ $\gamma_{k S}^{t}(G) \leq n-1$, we have to determine the maximum of the function $g$ on the interval $I: 2 \leq x \leq n-1$. It is easy to see that

$$
\begin{aligned}
\max _{x \in I}\{g(x)\} & =\max \{g(2), g(n-1)\} \\
& =\max \left\{2+\frac{n}{2}, n-1+\frac{n}{n-1}\right\} \\
& =n-1+\frac{n}{n-1}<n+1,
\end{aligned}
$$

and we obtain $\gamma_{k S}^{t}(G)+d_{k S}^{t}(G) \leq n$. This completes the proof.

Corollary 7. Let $G$ be a graph of order $n$ and let $k \geq 1$ be an integer. If $\min \left\{\gamma_{k S}^{t}(G), d_{k S}^{t}(G)\right\} \geq 2$, then

$$
\gamma_{k S}^{t}(G)+d_{k S}^{t}(G) \leq \frac{n}{2}+2
$$


Proof. Since $\min \left\{\gamma_{k S}^{t}(G), d_{k S}^{t}(G)\right\} \geq 2$, it follows by Theorem 4 that $2 \leq$ $d_{k S}^{t}(G) \leq \frac{n}{2}$. By (4) and the fact that the maximum of $g(x)=x+n / x$ on the interval $2 \leq x \leq n / 2$ is $g(2)=g(n / 2)$, we see that

$$
\gamma_{k S}^{t}(G)+d_{k S}^{t}(G) \leq d_{k S}^{t}(G)+\frac{n}{d_{k S}^{t}(G)} \leq \frac{n}{2}+2 .
$$

Observation 1 shows that Corollary 7 is no longer true if $\min \left\{\gamma_{k S}^{t}(G)\right.$, $\left.d_{k S}^{t}(G)\right\}=1$.

Theorem 8. The signed total $k$-domatic number of a graph is an odd integer.

Proof. Let $G$ be a graph, and suppose that $d=d_{k S}^{t}(G)$ is even. Let $\left\{f_{1}, f_{2}, \ldots\right.$, $\left.f_{d}\right\}$ be the corresponding signed total $k$-dominating family of $G$. If $u \in V(G)$, then $\sum_{i=1}^{d} f_{i}(u) \leq 1$. But on the left-hand side of this inequality, a sum of an even number of odd summands occurs. Therefore it is an even number, and we obtain $\sum_{i=1}^{d} f_{i}(u) \leq 0$ for each $u \in V(G)$. This forces

$$
\begin{aligned}
d & =\sum_{i=1}^{d} 1 \\
& \leq \sum_{i=1}^{d}\left(\frac{1}{k} \sum_{u \in N(v)} f_{i}(u)\right) \\
& =\frac{1}{k} \sum_{u \in N(v)} \sum_{i=1}^{d} f_{i}(u) \\
& \leq 0,
\end{aligned}
$$

which is a contradiction.

Theorem 9. Let $G$ be a graph and $v \in V(G)$. Then

$$
d_{k S}^{t}(G) \leq \begin{cases}\frac{\operatorname{deg}(v)}{k} & \text { if } \quad \operatorname{deg}(v) \equiv k \quad(\bmod 2) \\ \frac{\operatorname{deg}(v)}{k+1} & \text { if } \quad \operatorname{deg}(v) \equiv k+1 \quad(\bmod 2) .\end{cases}
$$

Moreover, if the equality holds, then for each function $f_{i}$ of a STkD family $\left\{f_{1}, f_{2}, \ldots, f_{d}\right\}$ and for every $u \in N(v), \sum_{u \in N(v)} f_{i}(u)=k$ if $\operatorname{deg}(v) \equiv k$ $(\bmod 2), \sum_{u \in N(v)} f_{i}(u)=k+1$ if $\operatorname{deg}(v) \equiv k+1(\bmod 2)$ and $\sum_{i=1}^{d} f_{i}(u)=1$.

Proof. Let $\left\{f_{1}, f_{2}, \ldots, f_{d}\right\}$ be a STkD family of $G$ such that $d=d_{k S}^{t}(G)$. If $\operatorname{deg}(v) \equiv k(\bmod 2)$, then

$$
\begin{aligned}
d & =\sum_{i=1}^{d} 1 \leq \sum_{i=1}^{d} \frac{1}{k} \sum_{u \in N(v)} f_{i}(u) \\
& =\frac{1}{k} \sum_{u \in N(v)} \sum_{i=1}^{d} f_{i}(u) \leq \frac{1}{k} \sum_{u \in N(v)} 1 \\
& =\frac{\operatorname{deg}(v)}{k} .
\end{aligned}
$$

Similarly, if $\operatorname{deg}(v) \equiv k+1(\bmod 2)$, then

$$
\begin{aligned}
d & =\sum_{i=1}^{d} 1 \leq \sum_{i=1}^{d} \frac{1}{k+1} \sum_{u \in N(v)} f_{i}(u) \\
& =\frac{1}{k+1} \sum_{u \in N(v)} \sum_{i=1}^{d} f_{i}(u) \leq \frac{1}{k+1} \sum_{u \in N(v)} 1 \\
& =\frac{\operatorname{deg}(v)}{k+1} .
\end{aligned}
$$


If $d_{k S}^{t}(G)=\frac{\operatorname{deg}(v)}{k}$ when $\operatorname{deg}(v) \equiv k(\bmod 2)$ or $d_{k S}^{t}(G)=\frac{\operatorname{deg}(v)}{k+1}$ when $\operatorname{deg}(v) \equiv k+1(\bmod 2)$, then the two inequalities occurring in the proof of each corresponding case become equalities, which gives the properties given in the statement.

Corollary 10. Let $G$ be a graph and $1 \leq k \leq \delta(G)$. Then

$$
d_{k S}^{t}(G) \leq \begin{cases}\frac{\delta(G)}{k} & \text { if } \quad \delta(G) \equiv k \quad(\bmod 2) \\ \frac{\delta(G)}{k+1} & \text { if } \quad \delta(G) \equiv k+1 \quad(\bmod 2) .\end{cases}
$$

Corollary 11. Let $1 \leq k \leq n \leq m$ be integers. Then

$$
d_{k S}^{t}\left(K_{n, m}\right) \leq\left\{\begin{array}{llll}
\min \left\{\frac{n}{k}, \frac{m}{k+1}\right\} & \text { if } n \equiv k \quad(\bmod 2) \text { and } m \neq n \quad(\bmod 2) \\
\frac{n}{k}{ }_{n} & \text { if } n \equiv k \quad(\bmod 2) \text { and } m \equiv n \quad(\bmod 2) \\
k+1 & \text { if } n \equiv k+1 \quad(\bmod 2) . &
\end{array}\right.
$$

The next two results are immediate consequences of Theorems 8 and 9 .

Corollary 12. For any tree $T, d_{S}^{t}(T)=1$.

Corollary 13. If $C_{n}$ is the cycle on $n$ vertices, then $d_{S}^{t}\left(C_{n}\right)=d_{2 S}^{t}\left(C_{n}\right)=1$.

Corollary 14. Let $G$ be a graph of order $n$. Then $\gamma_{k S}^{t}(G)+d_{k S}^{t}(G)=n+1$ if and only if $k \leq \delta(G) \leq k+1$ and for each $v \in V(G)$ there exists a vertex $u \in N(v)$ such that $\operatorname{deg}(u)=k$ or $\operatorname{deg}(u)=k+1$.

Proof. If $k \leq \delta(G) \leq k+1$ and for each $v \in V(G)$ there exists a vertex $u \in N(v)$ such that $\operatorname{deg}(u)=k$ or $\operatorname{deg}(u)=k+1$, then $\gamma_{k S}^{t}(G)=n$ by Observation 2 . Hence, $d_{k S}(G)=1$ and the result follows.

Conversely, let $\gamma_{k S}^{t}(G)+d_{k S}^{t}(G)=n+1$. The result is obviously true for $n=$ 2,3 . Assume $n \geq 4$. By Corollary 7, we may assume $\min \left\{\gamma_{k S}^{t}(G), d_{k S}^{t}(G)\right\}=1$. If $\gamma_{k S}^{t}(G)=1$, then $d_{k S}^{t}(G)=n$, which is a contradiction by Theorem 9 . If $d_{k S}^{t}(G)=1$, then $\gamma_{k S}^{t}(G)=n$ and the result follows by Observation 2 .

Theorem 15. For every graph $G$ of order $n$ and $1 \leq k \leq \min \{\delta(G), \delta(\bar{G})\}$,

$$
d_{k S}^{t}(G)+d_{k S}^{t}(\bar{G}) \leq \frac{n-1}{k}
$$

and equality in (6) implies that $G$ is a regular graph.

Proof. Since $\delta(G)+\delta(\bar{G}) \leq n-1$, by Corollary 10

$$
d_{k S}(G)+d_{k S}(\bar{G}) \leq \frac{\delta(G)}{k}+\frac{\delta(\bar{G})}{k} \leq \frac{n-1}{k} .
$$

If $G$ is not regular, then $\delta(G)+\delta(\bar{G}) \leq n-2$, hence $d_{k S}^{t}(G)+d_{k S}^{t}(\bar{G}) \leq \frac{n-2}{k}$. 


\section{The signed total $k$-domatic number of complete graphs}

In this section, we determine the value of signed total $k$-domatic number of a complete graph. First we determine the signed total $k$-domination number of $K_{n}$.

Lemma 16 ([4]). For $n \geq 2$,

$$
\gamma_{k S}^{t}\left(K_{n}\right)= \begin{cases}k+2 & n \equiv k \quad(\bmod 2) \\ k+1 & n \equiv k+1 \quad(\bmod 2)\end{cases}
$$

Proof. Assume $V\left(K_{n}\right)=\left\{v_{1}, \ldots, v_{n}\right\}$ is the vertex set of $K_{n}$. Suppose that $f$ is a signed total $k$-dominating function of $K_{n}$ and $f(v)=1$ for some $v \in V(G)$. If $n \equiv k(\bmod 2)$, then $f(N(v)) \geq k+1$ and hence, $f(V(G))=f(v)+f(N(v)) \geq$ $k+2$. Thus $\gamma_{k S}^{t}\left(K_{n}\right) \geq k+2$. Now define $f: V\left(K_{n}\right) \rightarrow\{-1,1\}$ by $f\left(v_{i}\right)=-1$ for $1 \leq i \leq \frac{n-k}{2}-1$ and $f\left(v_{i}\right)=1$ when $\frac{n-k}{2} \leq i \leq n$. It is easy to see that $f$ is a signed total $k$-dominating function on $K_{n}$ with $f\left(V\left(K_{n}\right)\right)=k+2$.

If $n \equiv k+1(\bmod 2)$, then $f(V(G))=f(v)+f(N(v)) \geq k+1$ and hence, $\gamma_{k S}^{t}\left(K_{n}\right) \geq k+1$. Now define $f: V\left(K_{n}\right) \rightarrow\{-1,1\}$ by $f\left(v_{i}\right)=-1$ for $1 \leq i \leq \frac{n-k-1}{2}$ and $f\left(v_{i}\right)=1$ when $\frac{n-k+1}{2} \leq i \leq n$. It is easy to see that $f$ is a signed total $k$-dominating function on $K_{n}$ with $f\left(V\left(K_{n}\right)\right)=k+1$. This completes the proof.

The next result is a generalization of Proposition A.

Theorem 17. For $n \geq 2$,

$$
d_{k S}^{t}\left(K_{n}\right)= \begin{cases}\left\lfloor\frac{n}{k+2}\right\rfloor & \text { if } n \equiv k \quad(\bmod 2) \text { and }\left\lfloor\frac{n}{k+2}\right\rfloor \text { is odd } \\ \left\lfloor\frac{n}{k+2}\right\rfloor-1 & \text { if } n \equiv k \quad(\bmod 2) \text { and }\left\lfloor\frac{n}{k+2}\right\rfloor \text { is even } \\ \left\lfloor\frac{n}{k+1}\right\rfloor & \text { if } n \equiv k+1 \quad(\bmod 2) \text { and }\left\lfloor\frac{n}{k+1}\right\rfloor \text { is odd } \\ \left\lfloor\frac{n}{k+1}\right\rfloor-1 & \text { if } n \equiv k+1 \quad(\bmod 2) \text { and }\left\lfloor\frac{n}{k+1}\right\rfloor \text { is even. }\end{cases}
$$

Proof. By Lemma 16 and Observation 1 we may assume $k \leq n-3$. Let $V\left(K_{n}\right)=\left\{x_{0}, x_{1}, \ldots, x_{n-1}\right\}$ be the vertex set of $K_{n}$. We consider two cases.

Case 1. $n \equiv k(\bmod 2)$. Suppose that $n=(k+2) q+r$, where $q$ is a positive integer and $0 \leq r \leq k+1$. By Lemma 16, $\gamma_{k S}^{t}\left(K_{n}\right)=k+2$. Hence, by Theorems 4 and $8, \bar{d}_{k S}^{t}\left(K_{n}\right) \leq q$ if $q$ is odd and $d_{k S}^{t}\left(K_{n}\right) \leq q-1$ if $q$ is even.

Subcase 1.1 $q$ is odd. Then $r$ is even. Define the functions $f_{1}, \ldots, f_{q}$ as follows.

$$
\begin{array}{lll}
f_{1}\left(x_{i}\right)=1 & \text { if } \quad 0 \leq i \leq \frac{(k+2)(q-1)}{2}+k+1, \\
f_{1}\left(x_{i}\right)=-1 & \text { if } \quad \frac{(k+2)(q-1)}{2}+k+2 \leq i \leq(k+2) q-1
\end{array}
$$

and for $2 \leq j \leq q$ and $0 \leq i \leq(k+2) q-1$,

$$
f_{j}\left(x_{i}\right)=f_{j-1}\left(x_{i+2(k+2)}\right) \text {, }
$$

where the sum is taken modulo $(k+2) q$. In addition, if $r>0$,

$$
f_{j}\left(x_{i}\right)=(-1)^{i+j} \text { for } 1 \leq j \leq q \text { and }(k+2) q \leq i \leq n-1 .
$$


It is easy to see that $f_{j}$ is a signed total $k$-dominating function of $K_{n}$ for each $1 \leq j \leq q$ and $\left\{f_{1}, \ldots, f_{q}\right\}$ is a signed total $k$-dominating family of $K_{n}$. Hence $d_{k S}^{t}\left(K_{n}\right) \geq q$. Therefore $d_{k S}^{t}\left(K_{n}\right)=q$, as desired.

Subcase $\mathbf{1 . 2} q$ is even. Then $r+k+2$ is even. Define the functions $f_{1}, \ldots, f_{q-1}$ as follows.

$$
\begin{array}{lll}
f_{1}\left(x_{i}\right)=1 & \text { if } & 0 \leq i \leq \frac{(k+2)(q-2)}{2}+k+1 \\
f_{1}\left(x_{i}\right)=-1 & \text { if } & \frac{(k+2)(q-2)}{2}+k+2 \leq i \leq(k+2)(q-1)-1
\end{array}
$$

and for $2 \leq j \leq q-1$ and $0 \leq i \leq(k+2)(q-1)-1$,

$$
f_{j}\left(x_{i}\right)=f_{j-1}\left(x_{i+2(k+2)}\right) \text {, }
$$

where the sum is taken modulo $(k+2)(q-1)$. In addition,

$$
f_{j}\left(x_{i}\right)=(-1)^{i+j} \text { for } 1 \leq j \leq q \text { and }(k+2)(q-1) \leq i \leq n-1 .
$$

It is easy to see that $f_{j}$ is a signed total $k$-dominating function of $K_{n}$ for each $1 \leq j \leq q-1$ and $\left\{f_{1}, \ldots, f_{q-1}\right\}$ is a signed total $k$-dominating family on $K_{n}$. Hence, $d_{k S}^{t}\left(K_{n}\right) \geq q-1$ and so $d_{k S}^{t}\left(K_{n}\right)=q-1$, as desired.

Case 2. $n \equiv k+1(\bmod 2)$. Suppose that $n=(k+1) q+r$, where $q$ is a positive integer and $0 \leq r \leq k$. By Lemma 16, $\gamma_{k S}^{t}\left(K_{n}\right)=k+1$. Hence, by Theorems 4 and $8, d_{k S}^{t}\left(K_{n}\right) \leq q$ if $q$ is odd and $d_{k S}^{t}\left(K_{n}\right) \leq q-1$ if $q$ is even.

Subcase 2.1 $q$ is odd. Then $r$ is even. Define the functions $f_{1}, \ldots, f_{q}$ as follows.

$$
\begin{array}{lll}
f_{1}\left(x_{i}\right)=1 & \text { if } \quad 0 \leq i \leq \frac{(k+1)(q-1)}{2}+k, \\
f_{1}\left(x_{i}\right)=-1 & \text { if } \quad \frac{(k+1)(q-1)}{2}+k+1 \leq i \leq(k+1) q-1
\end{array}
$$

and for $2 \leq j \leq q$ and $0 \leq i \leq(k+1) q-1$,

$$
f_{j}\left(x_{i}\right)=f_{j-1}\left(x_{i+2(k+1)}\right),
$$

where the sum is taken modulo $(k+1) q$. In addition, if $r>0$,

$$
f_{j}\left(x_{i}\right)=(-1)^{i+j} \text { for } 1 \leq j \leq q \text { and }(k+1) q \leq i \leq n-1 .
$$

It is easy to see that $f_{j}$ is a signed total $k$-dominating function of $K_{n}$ for each $1 \leq j \leq q$ and $\left\{f_{1}, \ldots, f_{q}\right\}$ is a signed total $k$-dominating family of $K_{n}$. Hence, $d_{k S}^{t}\left(K_{n}\right) \geq q$. Therefore $d_{k S}^{t}\left(K_{n}\right)=q$, as desired.

Subcase 2.2 $q$ is even. Then $r+k+1$ is even. Define the functions $f_{1}, \ldots, f_{q-1}$ as follows.

$$
\begin{array}{lll}
f_{1}\left(x_{i}\right)=1 & \text { if } & 0 \leq i \leq \frac{(k+1)(q-2)}{2}+k \\
f_{1}\left(x_{i}\right)=-1 & \text { if } & \frac{(k+1)(q-2)}{2}+k+1 \leq i \leq(k+1)(q-1)-1
\end{array}
$$

and for $2 \leq j \leq q-1$ and $0 \leq i \leq(k+1)(q-1)-1$,

$$
f_{j}\left(x_{i}\right)=f_{j-1}\left(x_{i+2(k+1)}\right),
$$

where the sum is taken modulo $(k+1)(q-1)$. In addition,

$$
f_{j}\left(x_{i}\right)=(-1)^{i+j} \text { for } 1 \leq j \leq q \text { and }(k+1)(q-1) \leq i \leq n-1 .
$$


It is easy to see that $f_{j}$ is a signed total $k$-dominating function of $K_{n}$ for each $1 \leq j \leq q-1$ and $\left\{f_{1}, \ldots, f_{q-1}\right\}$ is a signed total $k$-dominating family of $K_{n}$. Hence, $d_{k S}^{t}\left(K_{n}\right) \geq q-1$ and so $d_{k S}^{t}\left(K_{n}\right)=q-1$, as desired.

\section{The signed total $k$-domatic number of $K_{n, m}$}

In this section, we first determine the signed total $k$-domatic numbers for complete bipartite graphs $K_{n, n}$. Then we use this result to find the signed total $k$-domatic numbers for complete bipartite graphs $K_{n, m}$. This generalizes Proposition B for $k \geq 1$.

Theorem 18. For $n \geq 1$,

$$
d_{k S}^{t}\left(K_{n, n}\right)= \begin{cases}\left\lfloor\frac{n}{k}\right\rfloor & \text { if } n \equiv k \quad(\bmod 2) \text { and }\left\lfloor\frac{n}{k}\right\rfloor \text { is odd, } \\ \left\lfloor\frac{n}{k}\right\rfloor-1 & \text { if } n \equiv k \quad(\bmod 2) \text { and }\left\lfloor\frac{n}{k}\right\rfloor \text { is even, } \\ \left\lfloor\frac{n}{k+1}\right\rfloor & \text { if } n \equiv k+1 \quad(\bmod 2) \text { and }\left\lfloor\frac{n}{k+1}\right\rfloor \text { is odd, } \\ \left\lfloor\frac{n}{k+1}\right\rfloor-1 & \text { if } n \equiv k+1 \quad(\bmod 2) \text { and }\left\lfloor\frac{n}{k+1}\right\rfloor \text { is even. }\end{cases}
$$

Proof. By Theorem 3 and Observation 1, we may assume $k \leq n-2$. Let $X=\left\{x_{0}, x_{1}, \ldots, x_{n-1}\right\}$ and $Y=\left\{y_{0}, y_{1}, \ldots, y_{n-1}\right\}$ be the partite sets of $K_{n, n}$. We consider two cases.

Case 1. $n \equiv k(\bmod 2)$. Suppose that $n=k q+r$, where $q$ is a positive integer and $0 \leq r<k$. By Theorem $3, \gamma_{k S}^{t}\left(K_{n, n}\right)=2 k$. Hence, by Theorems 4 and 8 , $d_{k S}^{t}\left(K_{n, n}\right) \leq q$ if $q$ is odd and $d_{k S}^{t}\left(K_{n, n}\right) \leq q-1$ if $q$ is even.

Subcase 1.1 $q$ is odd. Then $r$ is even. Define the functions $f_{1}, \ldots, f_{q}$ as follows.

$$
\begin{array}{lll}
f_{1}\left(x_{i}\right)=f_{1}\left(y_{i}\right)=1 & \text { if } & 0 \leq i \leq \frac{k(q-1)}{2}+k-1 \\
f_{1}\left(x_{i}\right)=f_{1}\left(y_{i}\right)=-1 & \text { if } & \frac{k(q-1)}{2}+k \leq i \leq k q-1
\end{array}
$$

and for $2 \leq j \leq q$ and $0 \leq i \leq k q-1$,

$$
f_{j}\left(x_{i}\right)=f_{j-1}\left(x_{i+2 k}\right) \text { and } f_{j}\left(y_{i}\right)=f_{j-1}\left(y_{i+2 k}\right),
$$

where the sum is taken modulo $k q$. In addition, if $r>0$,

$$
f_{j}\left(x_{i}\right)=f_{j}\left(y_{i}\right)=(-1)^{i+j} \text { for } 1 \leq j \leq q \text { and } k q \leq i \leq n-1 .
$$

It is easy to see that $f_{j}$ is a signed total $k$-dominating function of $K_{n, n}$ for each $1 \leq j \leq q$ and $\left\{f_{1}, \ldots, f_{q}\right\}$ is a signed total $k$-dominating family of $K_{n, n}$. Hence $d_{k S}^{t}\left(K_{n, n}\right) \geq q$. Therefore $d_{k S}^{t}\left(K_{n, n}\right)=q$, as desired.

Subcase 1.2 $q$ is even. Then $r+k$ is even. Define the functions $f_{1}, \ldots, f_{q-1}$ as follows.

$$
\begin{array}{lll}
f_{1}\left(x_{i}\right)=f_{1}\left(y_{i}\right)=1 & \text { if } & 0 \leq i \leq \frac{k(q-2)}{2}+k-1 \\
f_{1}\left(x_{i}\right)=f_{1}\left(y_{i}\right)=-1 & \text { if } & \frac{k(q-2)}{2}+k \leq i \leq k(q-1)-1
\end{array}
$$

and for $2 \leq j \leq q-1$ and $0 \leq i \leq k(q-1)-1$,

$$
f_{j}\left(x_{i}\right)=f_{j-1}\left(x_{i+2 k}\right) \text { and } f_{j}\left(y_{i}\right)=f_{j-1}\left(y_{i+2 k}\right) \text {, }
$$


where the sum is taken modulo $k(q-1)$. In addition,

$$
f_{j}\left(x_{i}\right)=f_{j}\left(y_{i}\right)=(-1)^{i+j} \text { for } 1 \leq j \leq q \text { and } k(q-1) \leq i \leq n-1 .
$$

It is easy to see that $f_{j}$ is a signed total $k$-dominating function of $K_{n, n}$ for each $1 \leq j \leq q-1$ and $\left\{f_{1}, \ldots, f_{q-1}\right\}$ is a signed total $k$-dominating family on $K_{n, n}$. Hence, $d_{k S}^{t}\left(K_{n, n}\right) \geq q-1$ and so $d_{k S}^{t}\left(K_{n, n}\right)=q-1$ as desired.

Case 2. $n \not \equiv k(\bmod 2)$. Then $n \equiv k+1(\bmod 2)$. Suppose that $n=$ $(k+1) q+r$, where $q$ is a positive integer and $0 \leq r \leq k$. By Theorem 3, $\gamma_{k S}^{t}\left(K_{n, n}\right)=2(k+1)$. Hence, by Theorems 4 and $8, d_{k S}^{t}\left(K_{n, n}\right) \leq q$ if $q$ is odd and $d_{k S}^{t}\left(K_{n, n}\right) \leq q-1$ if $q$ is even.

Subcase 2.1 $q$ is odd. Then $r$ is even. Define the functions $f_{1}, \ldots, f_{q}$ as follows.

$$
\begin{array}{lll}
f_{1}\left(x_{i}\right)=f_{1}\left(y_{i}\right)=1 & \text { if } \quad & 0 \leq i \leq \frac{(k+1)(q-1)}{2}+k \\
f_{1}\left(x_{i}\right)=f_{1}\left(y_{i}\right)=-1 & \text { if } \quad & \frac{(k+1)(q-1)}{2}+k+1 \leq i \leq(k+1) q-1
\end{array}
$$

and for $2 \leq j \leq q$ and $0 \leq i \leq(k+1) q-1$,

$$
f_{j}\left(x_{i}\right)=f_{j-1}\left(x_{i+2(k+1)}\right) \text { and } f_{j}\left(y_{i}\right)=f_{j-1}\left(y_{i+2(k+1)}\right),
$$

where the sum is taken modulo $(k+1) q$. In addition, if $r>0$,

$$
f_{j}\left(x_{i}\right)=f_{j}\left(y_{i}\right)=(-1)^{i+j} \text { for } 1 \leq j \leq q \text { and }(k+1) q \leq i \leq n-1 .
$$

It is easy to see that $f_{j}$ is a signed total $k$-dominating function of $K_{n, n}$ for each $1 \leq j \leq q$ and $\left\{f_{1}, \ldots, f_{q}\right\}$ is a signed total $k$-dominating family of $K_{n, n}$. Hence, $d_{k S}^{t}\left(K_{n, n}\right) \geq q$. Therefore $d_{k S}^{t}\left(K_{n, n}\right)=q$, as desired.

Subcase 2.2 $q$ is even. Then $r+k+1$ is even. Define the functions $f_{1}, \ldots, f_{q-1}$ as follows.

$$
\begin{array}{lll}
f_{1}\left(x_{i}\right)=f_{1}\left(y_{i}\right)=1 & \text { if } & 0 \leq i \leq \frac{(k+1)(q-2)}{2}+k, \\
f_{1}\left(x_{i}\right)=f_{1}\left(y_{i}\right)=-1 & \text { if } & \frac{(k+1)(q-2)}{2}+k+1 \leq i \leq(k+1)(q-1)-1
\end{array}
$$

and for $2 \leq j \leq q-1$ and $0 \leq i \leq(k+1)(q-1)-1$,

$$
f_{j}\left(x_{i}\right)=f_{j-1}\left(x_{i+2(k+1)}\right) \text { and } f_{j}\left(y_{i}\right)=f_{j-1}\left(y_{i+2(k+1)}\right) \text {, }
$$

where the sum is taken modulo $(k+1)(q-1)$. In addition,

$$
f_{j}\left(x_{i}\right)=f_{j}\left(y_{i}\right)=(-1)^{i+j} \text { for } 1 \leq j \leq q \text { and }(k+1)(q-1) \leq i \leq n-1 .
$$

It is easy to see that $f_{j}$ is a signed total $k$-dominating function of $K_{n, n}$ for each $1 \leq j \leq q-1$ and $\left\{f_{1}, \ldots, f_{q-1}\right\}$ is a signed total $k$-dominating family of $K_{n, n}$. Hence, $d_{k S}^{t}\left(K_{n, n}\right) \geq q-1$ and so $d_{k S}^{t}\left(K_{n, n}\right)=q-1$, as desired.

Now we are ready to prove the main theorem of this section.

Theorem 19. Let $1 \leq k \leq n \leq m$ be integers. If $m \equiv n(\bmod 2)$, then $d_{k S}^{t}\left(K_{n, m}\right)=d_{k S}^{t}\left(K_{n, n}\right)$. If $m \not \equiv n(\bmod 2)$, then

$$
d_{k S}^{t}\left(K_{n, m}\right)
$$




$$
=\left\{\begin{array}{lll}
\left\lfloor\frac{n}{k}\right\rfloor & \text { if } n \equiv k \quad(\bmod 2),\left\lfloor\frac{n}{k}\right\rfloor \leq\left\lfloor\frac{m}{k+1}\right\rfloor \text { and }\left\lfloor\frac{n}{k}\right\rfloor \text { is odd, } \\
\left\lfloor\frac{n}{k}\right\rfloor-1 & \text { if } n \equiv k \quad(\bmod 2),\left\lfloor\frac{n}{k}\right\rfloor \leq\left\lfloor\frac{m}{k+1}\right\rfloor \text { and }\left\lfloor\frac{n}{k}\right\rfloor \text { is even, } \\
\left\lfloor\frac{m}{k+1}\right\rfloor & \text { if } n \equiv k \quad(\bmod 2),\left\lfloor\frac{n}{k}\right\rfloor \geq\left\lfloor\frac{m}{k+1}\right\rfloor \text { and }\left\lfloor\frac{m}{k+1}\right\rfloor \text { is odd, } \\
\left\lfloor\frac{m}{k+1}\right\rfloor-1 & \text { if } n \equiv k \quad(\bmod 2),\left\lfloor\frac{n}{k}\right\rfloor \geq\left\lfloor\frac{m}{k+1}\right\rfloor \text { and }\left\lfloor\frac{m}{k+1}\right\rfloor \text { is even, } \\
\left\lfloor\frac{n}{k+1}\right\rfloor & \text { if } n \equiv k+1 \quad(\bmod 2) \text { and }\left\lfloor\frac{n}{k+1}\right\rfloor \text { is odd, } \\
\left\lfloor\frac{n}{k+1}\right\rfloor-1 & \text { if } n \equiv k+1 \quad(\bmod 2) \text { and }\left\lfloor\frac{n}{k+1}\right\rfloor \text { is even. }
\end{array}\right.
$$

Proof. First let $m \equiv n(\bmod 2)$. By Corollary $11, d_{k S}^{t}\left(K_{n, m}\right) \leq \frac{n}{k}$ if $n \equiv k$ $(\bmod 2)$ and $d_{k S}^{t}\left(K_{n, m}\right) \leq \frac{n}{k+1}$ if $n \equiv k+1(\bmod 2)$. Hence, by Theorem $18, d_{k S}^{t}\left(K_{n, m}\right) \leq d_{k S}^{t}\left(K_{n, n}\right)$. Let $\left\{f_{1}, f_{2}, \ldots, f_{d}\right\}$ be a STkD family of $K_{n, n}$ and $d=d_{k S}^{t}\left(K_{n, n}\right)$. We extend this family to a STkD family of $K_{n, m}$. Add the new vertices $\left\{w_{1}, w_{2}, \ldots, w_{m-n}\right\}$ to a partite set of $K_{n, n}$ and join each $w_{i}$, $1 \leq i \leq m-n$, to every vertex in the other partite set of $K_{n, n}$ to obtain a $K_{n, m}$. Define $f_{j}^{*}: K_{n, m} \rightarrow\{-1,1\}$ as follows: $f_{j}^{*}(v)=f_{j}(v)$ if $v \in V\left(K_{n, n}\right)$ and $f_{j}\left(w_{i}\right)=(-1)^{i+j}$ for $1 \leq i \leq m-n$ and $1 \leq j \leq d$. Since $m \equiv n$ (mod 2), $\left\{f_{1}^{*}, f_{2}^{*}, \ldots, f_{d}^{*}\right\}$ is a STkD family of $K_{n, m}$, so $d_{k S}^{t}\left(K_{n, m}\right) \geq d$, and hence $d_{k S}^{t}\left(K_{n, m}\right)=d_{k S}^{t}\left(K_{n, n}\right)$.

Now assume $m \not \equiv n(\bmod 2)$. Let $X=\left\{x_{0}, x_{1}, \ldots, x_{n-1}\right\}$ and $Y=$ $\left\{y_{0}, y_{1}, \ldots, y_{m-1}\right\}$ be the partite sets of $K_{n, m}$. We consider two cases.

Case 1. $n \equiv k(\bmod 2)$. Then $m \equiv k+1(\bmod 2)$. Assume $n=k q_{1}+r_{1}$, where $0 \leq r_{1} \leq k-1$, and $m=(k+1) q_{2}+r_{2}$, where $0 \leq r_{2} \leq k$.

Subcase $1.1 q_{1} \leq q_{2}$. Then $d_{k S}^{t}\left(K_{n, m}\right) \leq q_{1}$ if $q_{1}$ is odd by Corollary 11, and $d_{k S}^{t}\left(K_{n, m}\right) \leq q_{1}-1$ if $q_{1}$ is even by Corollary 11 and Theorem 8 . Let $q_{2}=q_{1}+s$ for some $s \geq 0$. Then

$$
m=(k+1)\left(q_{1}+s\right)+r_{2}=(k+1) q_{1}+s(k+1)+r_{2} .
$$

If $q_{1}$ is odd, by assumptions, $r_{1}$ and $s(k+1)+r_{2}$ are both even.

Define the functions $f_{1}, \ldots, f_{q_{1}}$ as follows.

$$
\begin{array}{lll}
f_{1}\left(x_{i}\right)=1 & \text { if } & 0 \leq i \leq \frac{k\left(q_{1}+1\right)}{2}-1, \\
f_{1}\left(x_{i}\right)=-1 & \text { if } & \frac{k\left(q_{1}+1\right)}{2} \leq i \leq k q_{1}-1, \\
f_{1}\left(y_{j}\right)=1 & \text { if } & 0 \leq j \leq \frac{(k+1)\left(q_{1}+1\right)}{2}-1, \\
f_{1}\left(y_{j}\right)=-1 & \text { if } & \frac{(k+1)\left(q_{1}+1\right)}{2} \leq j \leq(k+1) q_{1}-1
\end{array}
$$

and for $2 \leq \ell \leq q_{1}, 0 \leq i \leq k q_{1}-1$ and $0 \leq j \leq(k+1) q_{1}-1$,

$$
f_{\ell}\left(x_{i}\right)=f_{\ell-1}\left(x_{i+2 k}\right) \text { and } f_{\ell}\left(y_{j}\right)=f_{\ell-1}\left(y_{j+2(k+1)}\right) \text {, }
$$

where the sum is taken modulo $k q_{1}$ if $i$ is involved, and modulo $(k+1) q_{1}$ if $j$ is involved. In addition, for $1 \leq \ell \leq q_{1}, k q_{1} \leq i \leq n-1$ and $(k+1) q_{1} \leq j \leq m-1$,

$$
f_{\ell}\left(x_{i}\right)=(-1)^{i+\ell} \text { and } f_{\ell}\left(y_{j}\right)=(-1)^{j+\ell} \text {. }
$$

It is straightforward to see that $\left\{f_{1}, \ldots, f_{q_{1}}\right\}$ is a signed total $k$-dominating family of $K_{n, m}$. Hence $d_{k S}^{t}\left(K_{n, m}\right) \geq q_{1}$. Therefore $d_{k S}^{t}\left(K_{n, m}\right)=q_{1}$, as desired.

Now let $q_{1}$ be even. We have $n=k\left(q_{1}-1\right)+k+r_{1}$ and

$$
m=(k+1)\left(q_{1}+s\right)+r_{2}=(k+1)\left(q_{1}-1\right)+(s+1)(k+1)+r_{2} .
$$


By assumptions, $k+r_{1}$ and $(s+1)(k+1)+r_{2}$ are both even. Define the functions $f_{1}, \ldots, f_{q_{1}-1}$ as follows.

$$
\begin{array}{lll}
f_{1}\left(x_{i}\right)=1 & \text { if } & 0 \leq i \leq \frac{k q_{1}}{2}-1 \\
f_{1}\left(x_{i}\right)=-1 & \text { if } & \frac{k q_{1}}{2} \leq i \leq k\left(q_{1}-1\right)-1, \\
f_{1}\left(y_{j}\right)=1 & \text { if } \quad & 0 \leq j \leq \frac{(k+1) q_{1}}{2}-1, \\
f_{1}\left(y_{j}\right)=-1 & \text { if } & \frac{(k+1) q_{1}}{2} \leq j \leq(k+1)\left(q_{1}-1\right)-1
\end{array}
$$

and for $2 \leq \ell \leq q_{1}-1,0 \leq i \leq k\left(q_{1}-1\right)-1$ and $0 \leq j \leq(k+1)\left(q_{1}-1\right)-1$,

$$
f_{\ell}\left(x_{i}\right)=f_{\ell-1}\left(x_{i+2 k}\right) \text { and } f_{\ell}\left(y_{j}\right)=f_{\ell-1}\left(y_{j+2(k+1)}\right),
$$

where the sum is taken modulo $k\left(q_{1}-1\right)$ if $i$ is involved, and modulo $(k+$ $1)\left(q_{1}-1\right)$ if $j$ is involved. In addition, for $1 \leq \ell \leq q_{1}-1, k\left(q_{1}-1\right) \leq i \leq n-1$ and $(k+1)\left(q_{1}-1\right) \leq j \leq m-1$,

$$
f_{\ell}\left(x_{i}\right)=(-1)^{i+\ell} \text { and } f_{\ell}\left(y_{j}\right)=(-1)^{j+\ell} .
$$

It is straightforward to see that $\left\{f_{1}, \ldots, f_{q_{1}-1}\right\}$ is a signed total $k$-dominating family of $K_{n, m}$. Hence $d_{k S}^{t}\left(K_{n, m}\right) \geq q_{1}-1$. Therefore $d_{k S}^{t}\left(K_{n, m}\right)=q_{1}-1$, as desired.

Subcase $1.2 q_{1}>q_{2}$. Then $d_{k S}^{t}\left(K_{n, m}\right) \leq q_{2}$ if $q_{2}$ is odd, by Corollary 11, and $d_{k S}^{t}\left(K_{n, m}\right) \leq q_{2}-1$ if $q_{2}$ is even, by Corollary 11 and Theorem 8 . Let $q_{1}=q_{2}+t$ for some $t \geq 0$. Then $n=k\left(q_{2}+t\right)+r_{1}=k q_{2}+k t+r_{1}$. If $q_{2}$ is odd, by assumptions, $r_{2}$ and $k t+r_{1}$ are both even. If $q_{2}$ is even, then $m=(k+1)\left(q_{2}-1\right)+k+1+r_{2}$ and $n=k\left(q_{2}+t\right)+r_{1}=k\left(q_{2}-1\right)+k(t+1)+r_{1}$. By assumptions, $k+1+r_{2}$ and $k(t+1)+r_{1}$ are both even. Hence, by an argument similar to that described in Subcase 1.1 we see that if $q_{2}$ is odd, $d_{k S}^{t}\left(K_{n, m}\right)=q_{2}$ and if $q_{2}$ is even, $d_{k S}^{t}\left(K_{n, m}\right)=q_{2}-1$.

Case 2. $n \equiv k+1(\bmod 2)$. Then $m \equiv k(\bmod 2)$. Let $n=(k+1) q+r$, where $0 \leq r \leq k$. Then $d_{k S}^{t}\left(K_{n, m}\right) \leq q$ if $q$ is odd by Corollary 11, and $d_{k S}^{t}\left(K_{n, m}\right) \leq q-1$ if $q$ is even by Corollary 11 and Theorem 8 . Let $m=n+s$, where $s \geq 0$. Then $m=k q+q+r+s$. If $q$ is odd, then $r$ and $q+r+s$ are both even. Define the functions $f_{1}, \ldots, f_{q}$ as follows.

$$
\begin{array}{lll}
f_{1}\left(x_{i}\right)=1 & \text { if } & 0 \leq i \leq \frac{(k+1)(q+1)}{2}-1, \\
f_{1}\left(x_{i}\right)=-1 & \text { if } & \frac{(k+1)(q+1)}{2} \leq i \leq(k+1) q-1, \\
f_{1}\left(y_{j}\right)=1 & \text { if } & 0 \leq j \leq \frac{k(q+1)}{2}-1, \\
f_{1}\left(y_{j}\right)=-1 & \text { if } & \frac{k(q+1)}{2} \leq j \leq k q-1
\end{array}
$$

and for $2 \leq \ell \leq q, 0 \leq i \leq(k+1) q-1$ and $0 \leq j \leq k q-1$,

$$
f_{\ell}\left(x_{i}\right)=f_{\ell-1}\left(x_{i+2(k+1)}\right) \text { and } f_{\ell}\left(y_{j}\right)=f_{\ell-1}\left(y_{j+2 k}\right) \text {, }
$$

where the sum is taken modulo $(k+1) q$ if $i$ is involved, and modulo $k q$ if $j$ is involved. In addition, for $1 \leq \ell \leq q,(k+1) q \leq i \leq n-1$ and $k q \leq j \leq m-1$,

$$
f_{\ell}\left(x_{i}\right)=(-1)^{i+\ell} \text { and } f_{\ell}\left(y_{j}\right)=(-1)^{j+\ell} \text {. }
$$


It is straightforward to see that $\left\{f_{1}, f_{2}, \ldots, f_{q}\right\}$ is a signed total $k$-dominating family of $K_{n, m}$. Hence $d_{k S}^{t}\left(K_{n, m}\right) \geq q$. Therefore $d_{k S}^{t}\left(K_{n, m}\right)=q$, as desired.

If $q$ is even, we write $n=(k+1)(q-1)+k+1+r$ and $m=k(q-1)+k+q+r+s$. By assumptions, $k+1+r$ and $k+q+r+s$ are both even. Define the functions $f_{1}, \ldots, f_{q-1}$ as follows.

$$
\begin{array}{lll}
f_{1}\left(x_{i}\right)=1 & \text { if } & 0 \leq i \leq \frac{(k+1) q}{2}-1 \\
f_{1}\left(x_{i}\right)=-1 & \text { if } & \frac{(k+1) q}{2} \leq i \leq(k+1)(q-1)-1, \\
f_{1}\left(y_{j}\right)=1 & \text { if } & 0 \leq j \leq \frac{k q}{2}-1 \\
f_{1}\left(y_{j}\right)=-1 & \text { if } & \frac{k q}{2} \leq j \leq k(q-1)-1
\end{array}
$$

and for $2 \leq \ell \leq q-1,0 \leq i \leq(k+1)(q-1)-1$ and $0 \leq j \leq k(q-1)-1$,

$$
f_{\ell}\left(x_{i}\right)=f_{\ell-1}\left(x_{i+2(k+1)}\right) \text { and } f_{\ell}\left(y_{j}\right)=f_{\ell-1}\left(y_{j+2 k}\right) \text {, }
$$

where the sum is taken modulo $(k+1)(q-1)$ if $i$ is involved, and modulo $k(q-1)$ if $j$ is involved. In addition, for $1 \leq \ell \leq q-1,(k+1)(q-1) \leq i \leq n-1$ and $k(q-1) \leq j \leq m-1$,

$$
f_{\ell}\left(x_{i}\right)=(-1)^{i+\ell} \text { and } f_{\ell}\left(y_{j}\right)=(-1)^{j+\ell} \text {. }
$$

It is straightforward to see that $\left\{f_{1}, f_{2}, \ldots, f_{q-1}\right\}$ is a signed total $k$-dominating family of $K_{n, m}$. Hence $d_{k S}^{t}\left(K_{n, m}\right) \geq q-1$. Therefore $d_{k S}^{t}\left(K_{n, m}\right)=q-1$, as desired.

Acknowledgement. The authors would like to thank the referee whose suggestions were most helpful in writing the final version of this paper.

\section{References}

[1] M. Guan and E. Shan, Signed total domatic number of a graph, J. Shanghai Univ. 12 (2008), no. 1, 31-34.

[2] M. A. Henning, Signed total domination in graphs, Discrete Math. 278 (2004), no. 1-3, 109-125.

[3] $\_$On the signed total domatic number of a graph, Ars Combin. 79 (2006), 277-288.

[4] C. P. Wang, The signed $k$-domination numbers in graphs, Ars Combin. (to appear).

[5] D. B. West, Introduction to Graph Theory, Prentice-Hall, Inc, 2000.

[6] B. Zelinka, Signed total domination number of a graph, Czechoslovak Math. J. 51(126) (2001), no. 2, 225-229.

\section{Abdollah Khodkar}

Department of Mathematics

University of West Georgia

Carrollton, GA 30118, USA

E-mail address: akhodkar@westga.edu

S. M. Sheikholeslami

Department of Mathematics

Azarbaijan University of Tarbiat Moallem

TABRIZ, I. R. IRAN

E-mail address: s.m.sheikholeslami@azaruniv.edu 